\title{
Lasers XUV collisionnels pompés par des lasers femtoseconde
}

\author{
S. Sebban, L.M. Upcraft, Ph. Balcou, M. Pittman, R. Haroutunian, G. Grillon, \\ C. Valentin, A. Rousse, J.P. Rousseau, L. Notebaert, D. Hulin, T. Mocek ${ }^{1}$, \\ B. Rus ${ }^{1}$, D. Ros ${ }^{2}$, A. Klisnick², A. Carillon ${ }^{2}$ et G. Jamelot ${ }^{2}$
}

\author{
Laboratoire d'Optique Appliquée, UMR 7639 du CNRS, 91761 Palaiseau cedex, France \\ 1 Institute of Physics, Academy of Sciences of the Czech Republic, Prague 8, Czech Republic \\ ${ }^{2}$ Laboratoire de Spectroscopie Atomique et lonique, UMR 8624 du CNRS, 91405 Orsay cedex, \\ France
}

\begin{abstract}
Résumé : Nous présentons de récents travaux sur la production de sources lasers XUV collisionels pompés par des impulsions laser femtoseconde. Le milieu amplificateur est un plasma créé d'ions multi-chargés et l'effet laser est observé sur les transitions $5 \mathrm{p}-5 \mathrm{~d}$ à $41,8 \mathrm{~nm}$ du xénon IX et $4 d-4 p$ du krypton IX à $32,8 \mathrm{~nm}$. L'influence des conditions d'irradiation et de densité du milieu lasant sur l'intensité de la source est présentée et discutée.
\end{abstract}

\section{INTRODUCTION}

Utiliser des lasers femtoseconde ( $\mathrm{fs}$ ) pour pomper des lasers XUV en schéma collisionnel a été proposé dès 1988 [1]. Il a fallu toutefois attendre 1995 pour qu'une forte amplification de la raie $5 \mathrm{~d}-5 \mathrm{p}$ du xénon palladiumoïde à $41,8 \mathrm{~nm}$ [2] soit observée en utilisant un laser de dimension modeste $(70 \mathrm{~mJ}$ en $50 \mathrm{fs})$. Ce travail expérimental, précédé d'études théoriques [3], ouvrait la voix à la réalisation de sources XUV monochromatiques et intenses, à haut taux de répétition et de dimension réduite. Toutefois, aucune confirmation de ce résultat n'a pu avoir lieu au cours des 5 années qui suivirent malgré de nombreuses tentatives à travers le monde. Ceci montrant que la physique de ce type de laser XUV n'était pas complètement maîtrisée. C'est en 2000 que ce résultat fut confirmé et amélioré au Laboratoire d'Optique Appliquée (LOA); une amplification saturée a été démontrée pour la première fois pour ce type de source [4]. Nous présenterons ici les résultats les plus marquants obtenus au LOA sur la production et la caractérisation de sources lasers XUV collisionnels pompés par des impulsions fs.

\section{DISPOSITIF EXPERIMENTAL}

Le milieu amplificateur est ici un plasma d'ion multichargés (Xe IX ou $\mathrm{Kr}$ IX). Les transitions laser sont respectivement $5 d-5 p$ et $4 d-4 p$ pour les ions Xe IX et $\mathrm{Kr}$ IX, à 41,8 et $32,8 \mathrm{~nm}$. Le schéma d'inversion de population est un schéma dit "collisionnel". Il fonctionne comme un laser à 3 niveaux. Le niveau supérieur de la transition est peuplé par excitations collisionnelles (collisions électrons-ions) à partir du niveau fondamental de l'ion huit fois chargé alors que le niveau inférieur se dépeuple rapidement vers le niveau fondamental par transitions radiatives. Pour favoriser les collisions et donc le pompage, le laser qui crée le plasma amplificateur est polarisé circulairement. Dans ces conditions des électrons plus énergétiques sont produits et favorisent de forts taux de pompage.

Le dispositif expérimental est décrit sur la figure 1. Le milieu amplificateur est produir en focalisant un laser Ti : saphir délivrant des impulsions de $1 \mathrm{~J}$ en 30 fs [5] dans une cellule de gaz à l'aide d'un miroir sphérique en incidence normale $(R=2 \mathrm{~m})$. Une colonne de plasma 
de quelques mm de long sur une cinquantaine de microns de diamètre est alors produite. Dans des conditions favorables de densité et température du plasma, le signal émergent de la colonne de plasma est essentiellement dominé par l'émission de la raie laser qui est émise dans l'axe du laser de pompe. Pour couper le laser de pompe, un filtre en aluminium est disposé sur le trajet optique des faisceaux. Le rayonnement est ensuite analysé à l'aide d'un spectromètre $X$ à transmission $(2000 \mathrm{t} / \mathrm{mm})$.

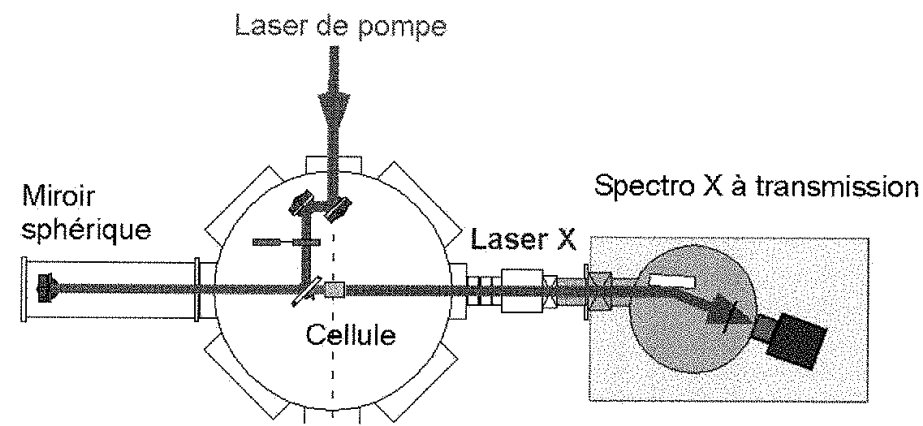

Figure 1. Description schématique du dispositif expérimental

\section{RESULTATS ET DISCUSSIONS}

\subsection{Spectres intégrés temporellement}

La figure 2 montre les spectres intégrés temporellement des plasmas de xénon et krypton. L'énergie du laser de pompe est respectivement de 500 et $800 \mathrm{~mJ}$ pour le xénon et le krypton, focalisée $1 \mathrm{~mm}$ après l'entrée de la cellule. Cette dernière fait 4 à $5 \mathrm{~mm}$ de long et contient 15 à 20 Torr de gaz. Nous voyons que les raies $5 p-5 d$ et $4 d-4 p$ dominent complètement les spectres d'émission du plasma, ce qui montre de façon manifeste un effet laser. Par ailleurs, nous voyons apparaître des raies satellites issues de la diffraction combinée de la raie laser XUV, à la fois sur le réseau et sur la grille support du réseau.

Grâce à une caméra CCD calibrée et une bonne connaissance de la transmission des filtres et de l'efficacité du spectromètre utilisé, nous avons estimé que le laser au xénon à 41.8 $\mathrm{nm}$ et le laser au krypton à $32.8 \mathrm{~nm}$ délivrent respectivement jusqu'à $5 \times 10^{9}$ et $2 \times 10^{9}$ photons par tir.
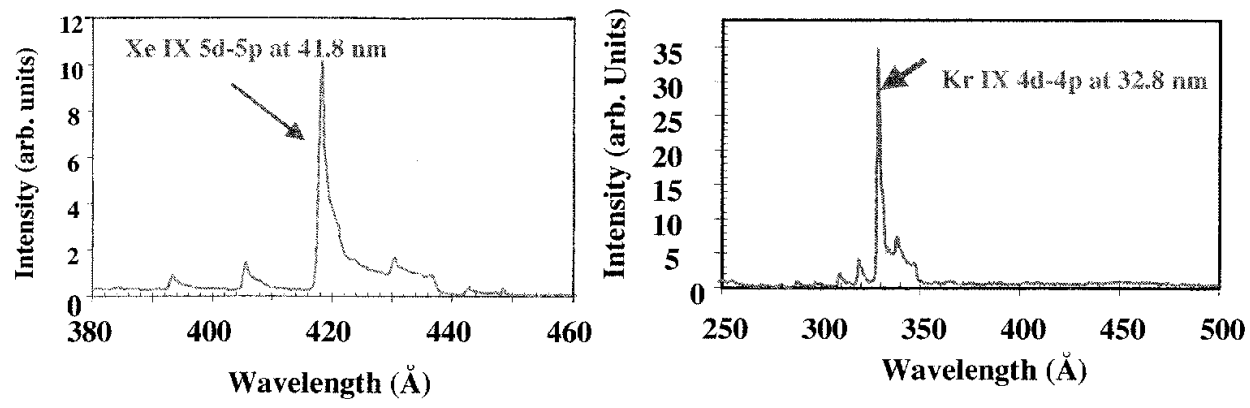

Figure 2. Spectres d'émission intégrés temporellement des plasmas de xénon et krypton 


\subsection{Influence de la pression du gaz sur l'émission laser}

Sur la figure 3, nous présentons l'intensité du signal à 41,8 et $32,8 \mathrm{~nm}$ en fonction de la pression du gaz dans la cellule. L'effet laser est maximum autour de 15-20 Torr pour les deux gaz utilisés et n'est effectif que dans une gamme relativement étroite de pression. Il faut noter que les calculs montrent que l'intensité laser XUV doit crôtre de façon monotone avec la pression car en augmentant la densité du gaz, donc la densité du plasma, nous favorisons aussi bien les processus collisionnels que nous augmentons l'intensité de saturation du milieu amplificateur. Les résultats présentés sur la figure 3 semblent montrer que le laser de pompe ne se propage pas correctement dans la cellule de gaz pour des pression supérieures à 15-20 torr. En effet, le profil d'intensité du laser de pompe (profil gaussien) dans la cellule est gaussien, ce qui implique que le gaz soit plus ionisé au centre que sur les bords de la tache focale. Ceci introduit un gradient d'indice optique qui joue le rôle d'une lentille divergente et modifie la propagation du laser dans la cellule de gaz. Le laser de pompe peut donc être réfracté, ce qui limite la longueur active du milieu amplificateur et donc limite l'effet laser. Cette réfraction par l'ionisation, qui est d'autant plus importante que le plasma est dense, est le processus le plus limitant dans l'amélioration des performances de ce type de source.

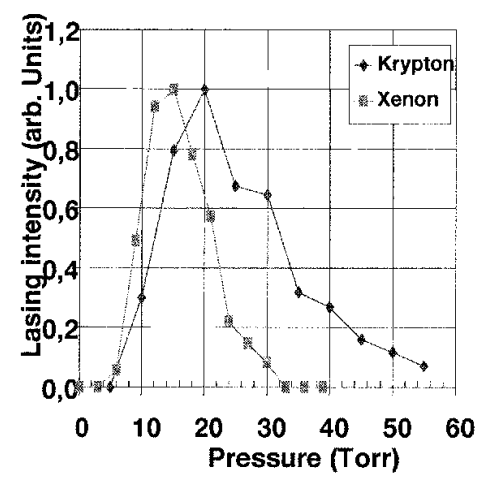

Figure 3. Dépendance en pression des raie du xénon IX à 41,8 et du krypton IX 32,8 nm

\subsection{Mesure du coefficient de gain}

Nous avons mesuré le coefficient de gain en variant la longueur de la cellule. La figure 4 montre l'intensité des raies $5 \mathrm{~d}-5 \mathrm{p}$ et $4 \mathrm{~d}-4 \mathrm{p}$ en fonction de la longueur du plasma. La pression du gaz est respectivement 15 et 20 Torr. Pour simplifier nous avons considéré que l'émissivité ne varie pas selon l'axe de propagation du laser de pompe et du laser XUV.

Un bon accord est obtenu pour des coefficients de gain $G_{0}=67 \pm 3 \mathrm{~cm}^{-1}$ pour la raie $5 \mathrm{~d}$ $5 p$ et $78 \pm 2 \mathrm{~cm}^{-1}$ pour la raie $4 \mathrm{p}-4 \mathrm{~d}$. Dans le cas du xénon, la saturation est atteinte pour une longueur de plasma de $2.2 \mathrm{~mm}$ ce qui donne un produit gain-longueur à la saturation de 15 . Ceci confirme bien que l'amplification est saturée. Dans le cas du krypton, la courbe montre la même tendance mais pour des longueurs de plasma bien supérieures. On obtient donc un produit gain-longueur de 27 ce qui n'est pas cohérent avec les intensités mesurées. Il semble que dans le cas du krypton, toute la longueur du plasma ne participe pas à l'amplification de la raie et que les longueurs du milieu, données dans la figure 4 , soient surestimées. Le milieu étant une cellule fermée, il est très difficile d'estimer de façon réaliste la longueur du milieu actif, ce qui limite la précision de ce type de mesure. 

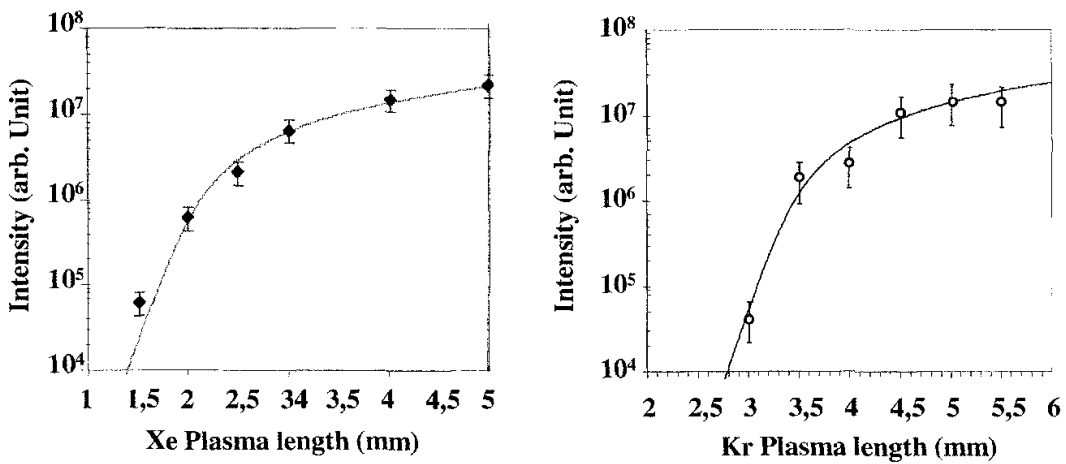

Figure 4. Intensité du signal laser en fonction de la longueur du plasma (raie $5 d-5 p$ du xénon IX à gauche et raie $4 \mathrm{~d}-4 \mathrm{p}$ du krypton IX à droite)

\section{CONCLUSION}

Ce travail montre l'émergence de nouvelles sources lasers XUV qui ont l'intérêt majeur d'émettre à $10 \mathrm{~Hz}$. Ces sources fonctionnent en régime saturé et délivrent jusqu'à $510^{9}$ photons par impulsion pour une durée de quelques picosecendes. Toutefois ces sources sont à améliorer. Il faudra dans un futur proche tenter de travailler à plus haute densité afin d'accroître sensiblement le nombre de photons par impulsion.

\section{Reférences}

[1] P.B. Corkum and N.H. Burnett, "Multiphoton ionization for the production of x-ray laser plasmas", in Short Wavelength Coherent Radiation, R.W. Falcone and J. Kirtz, Eds., Vol. 2 of OSA Proceedings Series (Optical Society of America, Washington, D.C., 1988) pp. 225-232

[2] B.E. Lemoff, C.L. Gordon, C.P.J. Barty and S.E. Harris., "Demonstration of a 10-Hz Femtosecond-Pulsedriven XUV Laser at $41.8 \mathrm{~nm}$ in Xe IX", Phys. Rev. Lett. 74, (1995) 1574-1577

[3]B.E. Lemoff, and, C.P.J. Barty, and S.E. Harris," Femtosecond-pulse-driven, electron excited XUV lasers in eigth-time ionized noble gas", Optics Letters 19, (1994) 569-571

[4].S. Sebban, R. Haroutunian, Ph. Bakou, G. Grillon, A. Rousse, S. Kazamias, T. Marin, J. P. Rousseau, L. Notebaert, M. Pittman, J. P. Chambaret, A. Antonetti, D. Hulin, D. Ros, A. Klisnick, A. Carillon, P. Jaeglé, G. Jamelot, and J.F Wyart, Phys. Rev. Lett, 86, (2001) 3004-3007

[5].M. Pittman, S. Ferré, J.P. Rousseau, L. Notebaert, J.P. Chambaret, and G. Chériaux, App. Phys B, 74 (2002) $529-535$ 